\title{
Development of glass microelectrodes pipette puller based on monitoring and controlling heating strength
}

\author{
Yuan Wang ${ }^{1,2, a}$, Li-Feng Fan ${ }^{1,3, b}$, Jian-Xu Wang, ${ }^{1,2, c}$, Yang Chen ${ }^{1,2, d}$, Lan \\ Huang $^{1,2,3, \mathrm{e}}$, Zhong-Yi Wang ${ }^{1,2,3}$ \\ ${ }^{1}$ College of Information and Electrical Engineering, China Agricultural University, Beijing \\ 100083,China; ${ }^{2}$ Modern Precision Agriculture System Integration Research Key Laboratory of \\ Ministry of Education, Beijing 100083, China; ${ }^{3}$ Key Laboratory of Agricultural information \\ acquisition technology (Beijing), Ministry of Agriculture, Beijing 100083, China; \\ a798711935@qq.com, ${ }^{b} 229670582 @ q q . c o m,{ }^{c} 1012443804 @ q q . c o m,{ }^{d}$ yanchcy@163.com, \\ ebiomed_hl@263.net, ${ }^{\mathrm{f}} w z y h l @$ cau.edu.cn
}

\begin{abstract}
The pulling of the glass microelectrode is a necessary technology in the electrophysiological measurement techniques. In order to better meet the requirements of electrophysiological experiments, we designed a multifunctional microelectrode puller, and gave the design of functional modules, and discussed its controlling effect. In this paper, we analyze the impact on the glass microelectrodes pulling which is brought by the heating strength based on the correspondence between the heating temperature and the heating wire resistance. Experimental results showed that the direct measurement of the heating temperature during the glass microelectrodes pulling process provided more direct control basis for the intelligent control, and provided a subtle improvement ideas for achieving precise pulling of the pipette puller.
\end{abstract}

Keywords: heating measurement, microelectrode, pipette puller, intelligent control

\section{Introduction}

A growing number of micro-electrode technologies show their superiority in the study of the electrochemical and electro analytical chemistry. Microelectrode usually refers to a very fine tip of the probe and is usually used in the measurement of both the intracellular and the extracellular domain[1]. The widely application of the microelectrode technology has a strong impetus to the research and development process of the electrophysiology[2-3]. The ion-selective microelectrode as the measurement of inside and outside biological cells and the new tools are widely used in biology, medicine and other disciplines[4]. SIET(self-referencing ion-selective microelectrode technique) is a new means of electrical physiology[5]. The microregion ion flow dynamic change information in the plant cell and the tissue can be obtained by the non-destructive detection technology. For the ion-selective microelectrodes isthe basic measurement tool, the producing of the glass microelectrode is a crucial aspect in the plant ion flow study. Therefore, pipette puller 
is the necessary equipment used in the electrophysiology laboratory for ion flow detection.

Nowadays, the drawing instrument on the market is divided into horizontal and vertical. The P97 series produced by the United States Sutter is the mainstream horizontal product, while the WD-1 Vertical Pullers is the most universal vertical one. Compared with the vertical puller, the electrode drawn by the horizontal puller is more symmetrical and repeatability. Aa a concequence, this study sets multifunctional micro-electrode pullers as the design goal, and takes some mainstream pullers as reference. In order to complete a glass microelectrodes instrument with convenient interactive interface, stable performance, monitoring and controllable heating intensity, both the software simulation and the system experiment are carried out to validate the feasibility of the whole design scheme.

\section{The overall design of the control circuits in the microelectrode puller}

According to the theory of the glass processing technology, the time, the intensity of the heating, and the cooling time are important indicators which determin the final performance parameters of the microelectrode ${ }^{[6]}$. Microelectrode pullers control circuit includes a microcontroller, heating control module, pulling control module, keyboard input interface and several functional units. The overall hardware module is shown in Fig. 1.

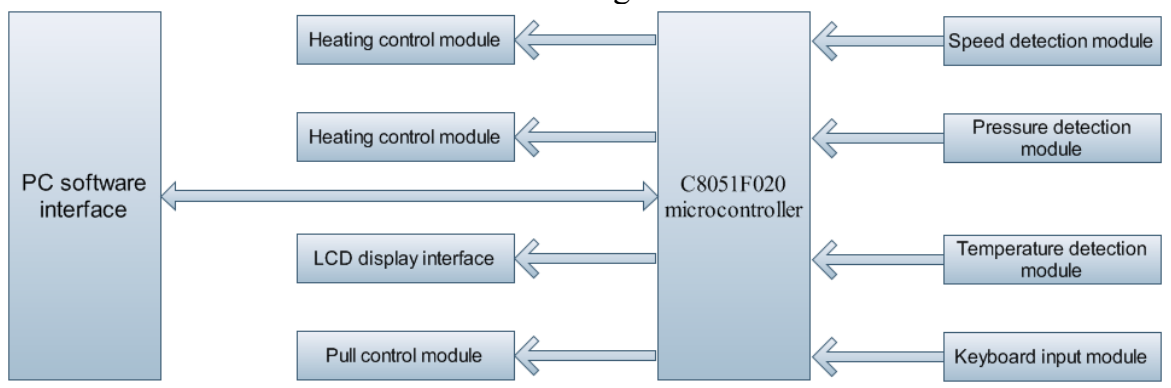

Fig.1. The overall hardware design block diagram

\subsection{Microcontrollers Selection}

The core controller of the control circuit is C8051F020 microcontroller. And its $\mathrm{ADC}$ and $\mathrm{DAC}$ can make analog and digital signal processing more easily. Two UART serial ports can be used for serial communication between PC and microcontroller. The large number of digital resources contained in the microcontroller can be allocated through priority crossbar compiler. Thus, it can ensure the flexibility of resource allocation. 


\subsection{Cooling Control Module}

This module includes pump and solenoid, and the regulation of the air flow can be achieved by their coordination. The adjustment is based on the pressure measured by the pressure sensor, and the microcontroller will determine a feedback adjustment instruction according to the results compared with the pre-set value.

\subsection{User Interface}

The users can control the instrument in two ways, including the PC software and the keyboard. The drawing parameters can be set through the matrix keyboard directly in front of the puller. The serial port can also realize the parameter setting and saving. Serial communication is realized by the USB bus converter chip CH341A which is produced by Nanjing Heng Qin. And the chip is used to provide asynchronous serial bus, printer port, parallel port, the usual 2-wire and 4-wire and other synchronous serial interface via USB. In addition to the control parameters passed to the microprocessor, the serial communication module can also help upload the drawing parameters and save the document. In addition, the hardware circuit also includes a LCD screen display circuit, using YLF12864H-1 models with character screen. The entire interface display includes the welcome screen, the menu interface and parameter settings sub-menu interface.

PC software interface and LCD screen are shown in Fig. 2 and Fig. 3:
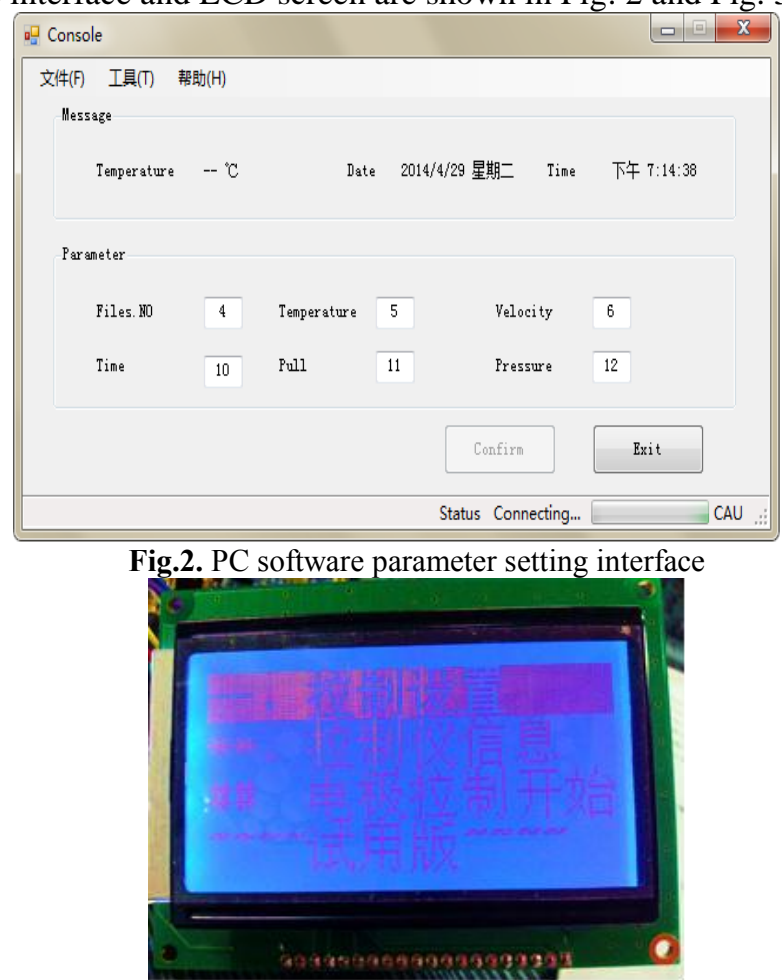


\section{Fig.3. LCD display interface 2.4. Heating control module and pull control module}

Heating control module and the pull control module is designed with FET which is a voltage controlled devices, namely, to achieve control of the drain current by the gatesource voltage. Heating control module uses AC-DC converter circuit, and its performance is also part of the circuit to do a more detailed experimental verification. Pull module selects the solenoid as the tension actuator. The size of the rally adjustment is realized through the current control in the solenoid. In the production process of glass, its melting, clarification, homogenizing, feeding, molding, and temperature system degradation process, are generally developed based on its corresponding viscosity. Therefore, glass viscosity variation has an important guiding significance on the control of the entire process of shaping glass. Speed detection value is mainly used to characterize the degree of softening of the glass tube blank. Therefore, it is an important indicator for the drawing process during the cooling, and heating control. Speed detection signals are mainly conditioned by amplifier circuits and filter circuits.

\section{Signal isolation solutions and experimental verification}

\subsection{Digital signal isolation}

Digital signal isolation methods are generally implemented by unidirectional digital signals, and the bidirectional digital signal isolation is achieved by employing two more elements. Common digital signal isolation methods are mainly (1) opt coupler, such as PC817, PS2501, 6N137, etc.; (2) ADI's magnetic isolation chip; (3) isolation [7-8] through the transformer. The design uses a si8422 single-chip and si8423 dualchannel digital isolators. Compared to traditional isolation techniques, ultra-low power digital isolation chip has advantages in the aspects of substantial data rate of CMOS devices, propagation delay, power, size, reliability. And it can remain stable under a wide range of temperatures and operating parameters during the life of the device so that it can be designed and widely used. What's more, the chip uses a narrow-body SOIC-8 package RoHS standard, which greatly saves installation space.

\subsection{Analog signal isolation method}

In a scenario which accurate measurement is needed, the isolation cost of the analog signal will be an important consideration factor. More common analog signal isolation methods include: (1) isolation amplifier; (2) the frequency to voltage converter combined with digital isolation; (3) the use of DA / AD combined with the digital isolation solution for analog signals sample recovery; (4) linear opt coupler[9]. The design uses a linear opt coupler HCNR201 model[10], and the circuit in Fig 4 is used for the two DA signals isolation. 


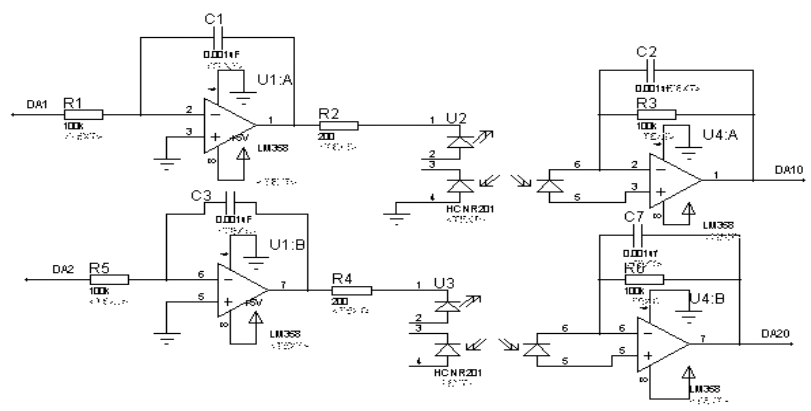

Fig.4. Analog signal isolation circuit schematics

The relationship diagram which is shown in Fig. 5 is obtained by measuring the analog input and output signal isolation circuit. Fig. 5 shows a high correlation coefficient between the input signal and the isolated signals. That is to say, the circuit secures a true effect.

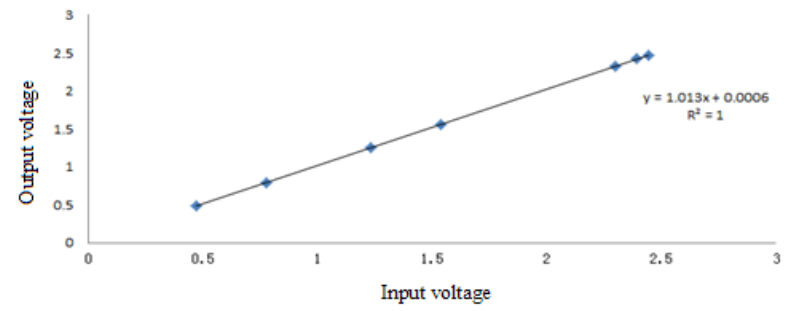

Fig.5. Signal isolation between input and output voltage linear analog schematic

\section{AC-DC converter circuit solutions and experimental verification}

\subsection{AC-DC converter circuit design}

Common controllers which are applied to help small current control large current mainly include: SCR, FET and relay. Taking power, on-time, efficiency, cost and other factors into consideration, our design chose FET as the control device. We designed a heating unit control module circuit with feedback regulation, and the circuit diagram is shown in Fig. 6. 


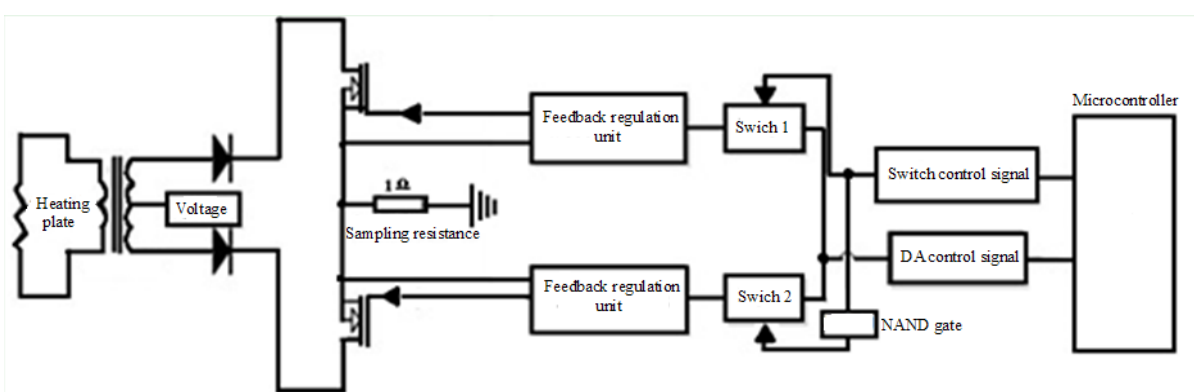

Fig.6. DC-AC converter circuit diagram

The scheme used DA value as a feedback to adjust the parameters so as to achieve the control on the size of the drain current in the FET. At the same time, the symmetrical on both sides conducts in a certain adjustable frequency alternately. In the two-way symmetrical circuit, output signal terminal was connected to a transformer, which was used to provide heating. In the regulation of the DA, both the two output signal amplitude and the extent of FET conduction changed accordingly. The user can achieve primary side voltage by adjusting the heating transformer. After connecting the heating wire to the secondary side of the transformer, the heating intensity can be adjusted according to a certain adjustable frequency.

\subsection{Circuit experimental verification}

For the heating program, we used the unit circuit verification to test the design, and measured the corresponding amplitude relationship with the DA control parameter value between the primary side and the secondary side of the transformer. We concluded that the voltage varied with the DA changes according to exprimental analysis. And the obtained results are shown in Fig. 7.

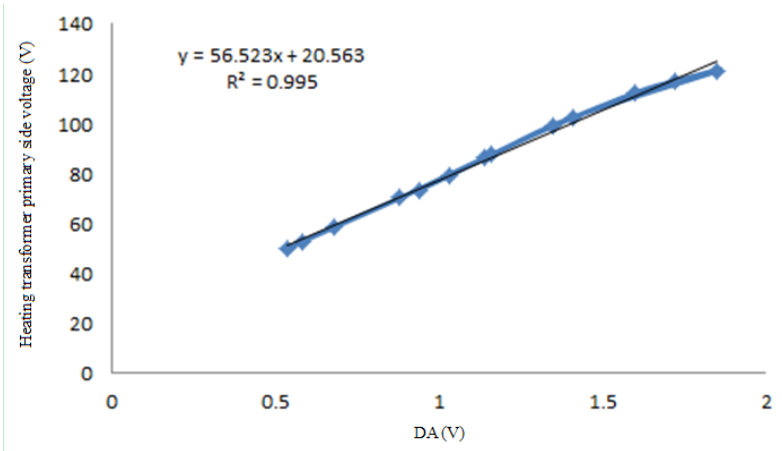

Fig.7. Heating transformer primary side voltage (V) measurement curve under DA (V) regulation 


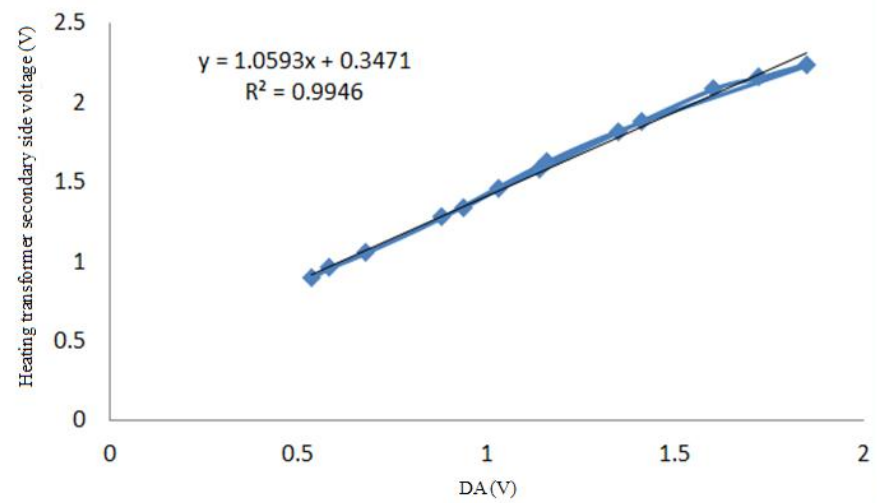

Fig.8. Heating transformer secondary side voltage DA (V) measurement curve under DA regulation.

Fig. 7 shows that the primary side of the transformer can achieve a range between $45 \mathrm{~V}$ to $125 \mathrm{~V}$ as the $\mathrm{DA}$ value ranges from $0.4 \mathrm{~V}$ to $2 \mathrm{~V}$. Besides, the secondary side Voltage control can be changed by adjusting the transformer turns ratio.

Fig. 8 shows the heating transformer secondary side voltage measurement curve under DA regulation. According to the transformer working theory, the secondary side varies according to the primary side .Thus, the secondary side voltage of the heating transformer will have a range as the DA regulates.

\subsection{Heating intensity detection module}

Heating intensity is the key factor during the entire drawing process. The constant resistance calculated by the voltage and current at both ends of the heating plate was used as the heating process indicator. And the heating voltage across the current measuring circuit is shown in Fig. 9.

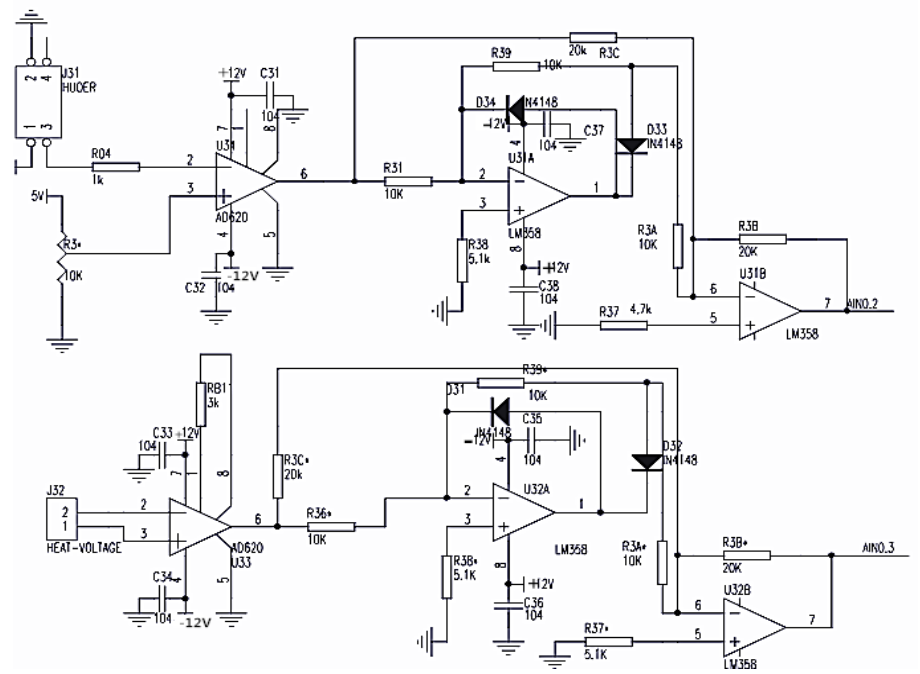

Fig.9. Heating intensity schematic 
By employing the nickel-chromium heating wire in the circuit, this experiment aimed to test the DA control status. The correspondence between the nickelchromium heating wire resistivity values and the DA regulation is shown in Fig. 10.

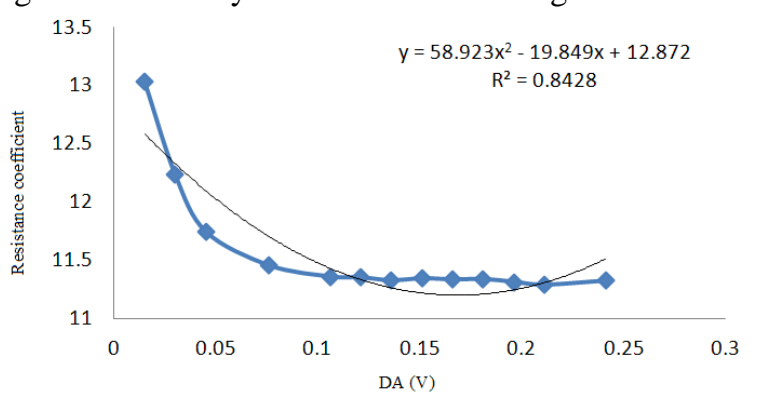

Fig.10. The relationship between nickel-chromium alloy DA (V) and the DA

According to the Fig. 10, the resistivity value of nichrome DA regulation is a quadratic function, and the correlation coefficient is 0.8428 . In the actual drawing process, the resistance is designed as the index of the heating strength.

\section{Proteus simulation}

Proteus EDA tool simulation software is published by the British Lab center electronics. Not only does it has the function that other EDA simulation has, but also it can cooperate with the Keil simulation and the peripheral devices. The proteus is the best simulation tool in the industry and microcontroller peripheral device so far ${ }^{[11]}$.

In order to validate the design effect of the module circuit, functional simulation for heating section and speed detection module by the proteus software are performed.

\subsection{Simulation results of heating module}

In simulation, we selected the amps, resistors, capacitors, switches and other components from the library in accordance with the circuit diagram and the simulation schematic which are shown in Fig. 11. By real-time monitoring the waveforms of input and output signal from the virtual oscilloscope, the control signals obtained on the both sides of the symmetrical circuit are shown in Fig.12. 


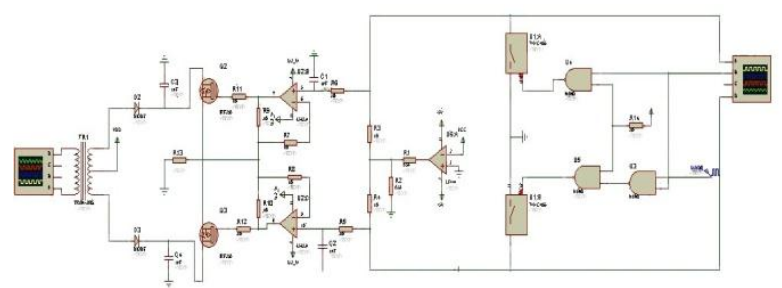

Fig.11. Heating unit circuit simulation schematic

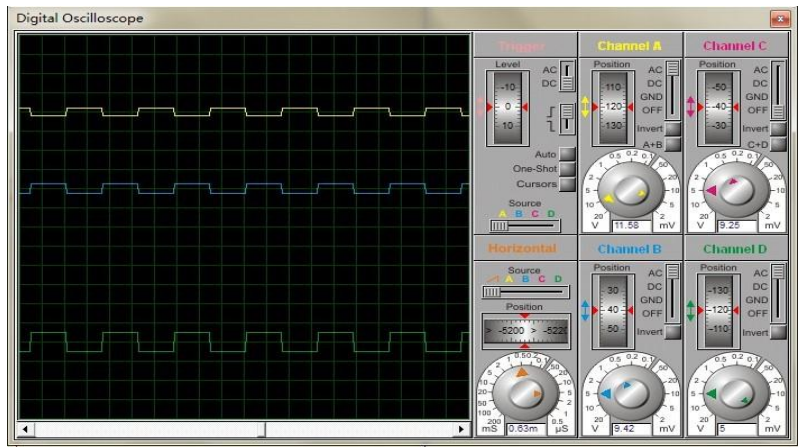

Fig.12. Proteus simulation run chart

As shown in the virtual oscilloscope, the two sides are sequentially turned on in a certain frequency (the frequency is adjustable), which is equal to the frequency of the transformer's AC signal on the primary side. Thus, the DA control signal functions in the two sides in turn, and will control the size of the drain current of the FET. Therefore, the amplitude of the AC voltage in the heating transformer's primary side is controllable.

\subsection{Simulation on the sensor signal conditioning circuit}

The speed sensor module and the pressure part used the similar signal conditioning circuit. Namely, the output voltage was turned into a certain range of the signal amplification by the AD620, and the active low-pass filter filtered out the invalid frequency. As the Fig. 13 shown, the three waveforms seen from the virtual oscilloscope are output voltage of the sensor, the amplified output signal and the waveform after AD620. 


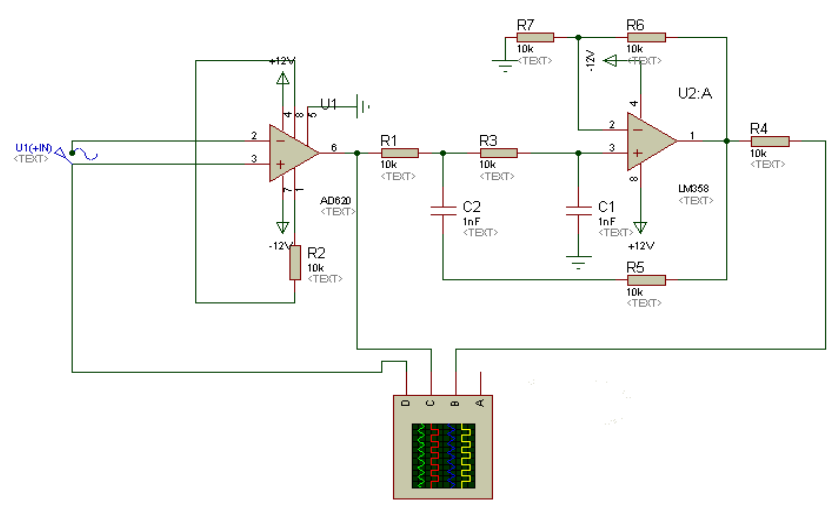

Fig.13. Pressure monitoring module simulation schematic

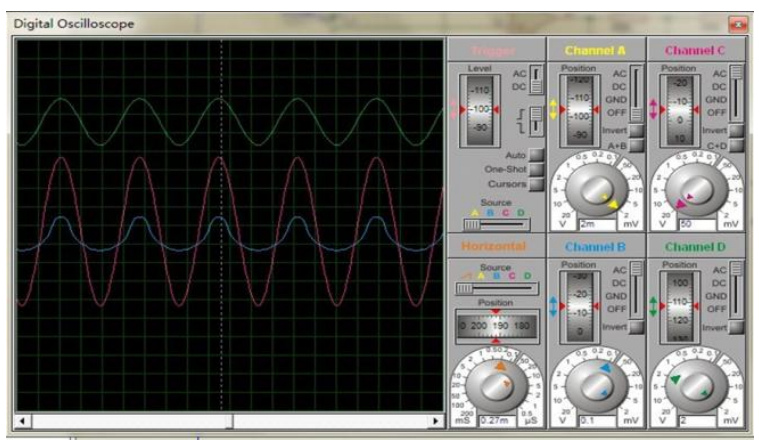

Figure.14. Virtual oscilloscope schematic emulation

In Fig. 14, the output signal of the speed sensor used $1 \mathrm{kHz}$ sine wave to simulate. Analyzing the waveform seen in the virtual oscilloscope, the sensor output voltage signal is amplified through AD620 by 5.94 times. However, the signals above $1 \mathrm{kHz}$ was filtered out by the low-pass filter circuit to ensure the effective output signals. Simulation results consisted with the design requirements, so the circuit design can meet the desired effect.

\section{Conclusion}

This paper provided a design idea on the control unit circuit of the multifunctional micro-electrode pullers. The performance verification results were given by experiments and proteus unit circuit simulation. Analysis conclusion showed that the functional modules can play the desired control effect. Isolation of the analog signal can meet the drawing instrument control signal transmission accuracy. Meanwhile, heating control circuit under the control of DA values can range $45 \mathrm{~V}$ - 
$125 \mathrm{~V}$ voltage regulator. The user can control the connected operating voltage and the current value performed on the load by changing the turns ratio between the heating transformer primary and secondary voltage.

The cooperation of every function module under the command of the microprocessor is the key factor. Future work will continue to make the control parts harmoniously work with the mechanical parts.

\section{Acknowledgements}

This is supported by National Key Scientific Instrument and Equipment Development Projects of China (2011YQ080052).

\section{References}

[1]. Ruihua Han, Dengping Gu. Microelectrode production situation and its application [J] Hebei Normal University (Natural Science), 1991, 2: 025(in Chinese with English abstract).

[2]. Glass A D M, Shaff J E, Kochian L V. Studies of the uptake of nitrate in barley IV. Electrophysiology[J]. Plant Physiology, 1992, 99(2): 456-463.

[3]. Henriksen G H, Bloom A J, Spanswick R M. Measurement of net fluxes of ammonium and nitrate at the surface of barley roots using ion-selective microelectrodes[J]. Plant Physiology, 1990, 93(1): 271-280.

[4].Xingang Wang, Han Ping Mao, Zuo Zhiyu.The application of ion-selective microelectrodes and patch clamp in detecting electrophysiology information [J]. Agricultural Mechanization Research, 2007 (10): 36-39(in Chinese with English abstract).

[5]. Xue Lin, Zhao Dongjie, Hou Peichen, et al. Test and preparation of microelectrode in applications of self-referencing ion electrode technique[J]. Transactions of the Chinese Society of Agricultural Engineering (Transactions of the CSAE), 2013, 29(16): 182-189. (in Chinese with English abstract)

[6]. Yu Guo. Glass products and mold design [M]. Chemical Industry Press Publishing Center of Materials Science and Engineering, 2003.(in Chinese)

[7]. Digital isolation techniques and technology. (http//www.chinaecnet.com). (in Chinese) [8]. Ho Jin. Selection and Application of Digital isolation devices [J]. Electronic Design \& Application World for Design and Application Engineers, 2008 (2): 57-61(in Chinese).

[9]. ZHANG B, WANG N. Analog Isolation Board Based on High-linearity Analog Optocoupler HCNR200 [J]. Instrumentation Technology, 2005, 5: 021.

[10]. Haibo Li, Hui Lin. Application in current sampling with delicate linear optocoupler HCNR200 [J]. World of electronic components, 2004 (11): 37-38(in Chinese).

[11]. Hong Liu, Futian Guo, Zhen-Xing Sun. The application of proteus simulation technology in single-chip microcomputer teaching[J]. Changchun Institute of Technology(Social Sciences Edition), 2007, 24 (3): 96-98(in Chinese with English abstract). 